\title{
What Diagrams Are Considered Useful for Solving Mathematical Word Problems in Japan?
}

\author{
Hiroaki Ayabe ${ }^{1(\bowtie)}$, Emmanuel Manalo $^{2}$, Mari Fukuda $^{3}$, and Norihiro Sadato ${ }^{1}$ \\ 1 National Institute for Physiological Sciences, Aichi, Japan \\ \{ayabe, sadato\}@nips.ac.jp \\ 2 Graduate School of Education, Kyoto University, Kyoto, Japan \\ manalo.emmanuel.3z@kyoto-u.ac.jp \\ 3 Graduate School of Education, The University of Tokyo, Tokyo, Japan \\ mari_fukuda@p.u-tokyo.ac.jp
}

\begin{abstract}
Previous studies have shown that diagram use is effective in mathematical word problem solving. However, they have also revealed that students manifest many problems in using diagrams for such purposes. A possible reason is an inadequacy in students' understanding of variations in types of problems and the corresponding kinds of diagrams appropriate to use. In the present study, a preliminary investigation was undertaken of how such correspondences between problem types and kinds of diagrams are represented in textbooks. One government-approved textbook series for elementary school level in Japan was examined for the types of mathematical word problems, and the kinds of diagrams presented with those problems. The analyses revealed significant differences in association between kinds of diagrams and types of problems. More concrete diagrams were included with problems involving change, combination, variation, and visualization of quantities; while number lines were more often used with comparison and variation problems. Tables and graphs corresponded to problems requiring organization of quantities; and more concrete diagrams and graphs to problems involving quantity visualization. These findings are considered in relation to the crucial role of textbooks and other teaching materials in facilitating strategy knowledge acquisition in students.
\end{abstract}

Keywords: Math education · Diagram use $\cdot$ Textbook research

\section{Introduction}

Mathematical word problem solving is commonly used in schools to cultivate students' abilities in applying mathematical knowledge and skills to everyday life. However, the complexity of cognitive processes demanded by this activity leads to a prevalence of difficulties in students [1]. Diagram use is generally considered one of the most effective strategies for promoting success in this kind of problem solving [2]. However, students tend not to use diagrams spontaneously, and even when they do use them, such use does not always lead to obtaining the correct solutions.

(C) The Author(s) 2021

A. Basu et al. (Eds.): Diagrams 2021, LNAI 12909, pp. 79-83, 2021.

https://doi.org/10.1007/978-3-030-86062-2_8 
One reason for the failure to solve despite the use of diagrams is a lack of correspondence between the problem schema/type (i.e., its structure and requirements) and the kind of diagram that students construct [3]. Word problems are included in the school curriculum to facilitate understanding of target learning contents, and diagrams are used as a way of "scaffolding" to make solving easier. However, inadequate attention is placed on teaching "appropriate diagram use" to match the problems given because the curriculum focuses on teaching "mathematical contents". Thus, much of the knowledge that students develop about diagram use remains implicit.

To address this problem, ways to effectively facilitate understanding of the correspondence between problem schema/type and appropriate diagrams need to be investigated. While it would be difficult to capture and evaluate the wide range of methods that teachers use to promote such understanding, examining how the textbooks might facilitate that understanding is much more manageable. In Japan, all schools use textbooks certified by the Ministry of Education in line with the national guidelines (issuance law of Japan). Examining such textbooks could provide valuable insights into how those correspondences are portrayed, which had not been examined in previous research. Therefore, this study aimed to clarify: (i) the types of word problems used in math textbooks, (ii) the kinds of diagrams deemed useful for solving those problems, and (iii) the correspondence between problems and diagrams.

\section{Method}

The textbooks used were one of six mathematics textbook series eligible for use in Japan at the elementary school level ( 6 books corresponding to Grades 1 to 6, each containing 13-19 chapters) [4]. Each chapter contains example problems with detailed explanations. The main purpose of those example problems is to teach the learning contents set by the national standard, assuming that children would be learning them for the first time. The present study focused on all the example problems that take the form of word problems, defined here as problems comprising two or more sentences with a background story, and which requires a mathematical solution. The word problems were examined and categorized according to their problem schema (i.e., type of word problem), and the kind of diagram deemed appropriate for solving them.

In line with previous studies [5], the word problems found were classified into one of six types: Change (e.g., Ken had 3 candies. Naomi gave him 5 more candies. How many candies does Ken have now?), Combine (e.g., Ken has 4 candies. Naomi has 3 candies. How many candies do they have altogether?), Compare (e.g., Ken has 4 candies. Naomi has 12 candies. How many times are Naomi's candies greater than Ken's?), Vary (e.g., Ken packs 5 candies in each box. How many boxes will he need to pack to fit 40 candies?), Organize (i.e., organizing data given to find an answer by using tables or graphs; e.g., The following data shows students' reading times in a month. What number appears the most often?), and Visualize (visualizing the conditions given in the problem with figures, graphs, tables, etc., to facilitate search for rules difficult to find based only on superficial details; e.g., There are 3 children in front of Ken, and 4 behind him. How many children are there in total?).

Diagrams were categorized into seven kinds: Pictures (images relating to the problem but conveying no quantitative information), Concrete diagrams (illustrations/pictures 


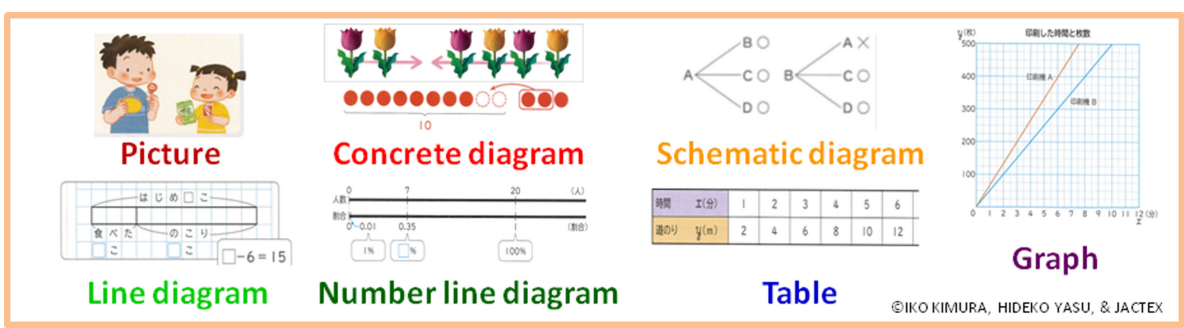

Fig. 1. Kinds of diagrams (with examples) used to categorize those found in the textbooks

with quantitative information, including semi-concrete representations of quantities using circles and other counters), Schematic diagram (arrows, lines, figures showing procedures and quantitative/functional relationships, including tree diagrams), Line diagrams (line or tape diagrams, segments of which indicate quantities to facilitate visual comparison), Number line diagrams (one or more lines arranged to show relationships between quantities, including proportional relationships), Tables (arrays of numbers, symbols, and words/letters), and Graphs (bar graphs, line graphs, statistical graphs such as histograms and dot plots, function graphs) (see Fig. 1). Coding for each textbook was independently undertaken by combinations of two of four school teachers, none of whom had any vested interest in the outcomes.

The reliability statistic between inter-raters indicated almost perfect agreement $($ Cohen's kappa $=0.84)$. Differences were settled through discussion.

\section{Results and Discussion}

There were 246 word problems found in the six textbooks (mean $=41.0 \pm 9.76$ ), and diagrams were included with all of them. The textbooks therefore clearly convey the importance of diagram use in problem solving. The numbers of word problems varied depending on grade level $\left(\chi^{2}{ }_{(5)}=11.61, p=.041\right)$ : lowest at Grade $1(n=25, p=$ $.003)$, and highest at Grade $5(n=54, p=.013)$. The Grade 1 textbook contains mostly simpler one-sentence problems, while the concept of proportionality is introduced in the Grade 5 textbook, which may partially explain those differences.

An analysis of residuals after Chi-squared tests identified proportional differences. Table 1 shows the frequencies with which each kind of diagram was included with each type of problem, with asterisks indicating those proportionally significantly higher or lower. In total, Concrete diagrams and Number line diagrams were found to be the most frequently used diagrams ( $p<.001$, for both). Concrete diagrams were often used with Change, Combine, Vary, and Visualize problems, suggesting that they are helpful in facilitating the four arithmetic operations and understanding of the problem scenario and contents. Number line diagrams were often used with Compare and Vary problems, suggesting that they are helpful when comparing measurement quantities and performing proportional calculations. More abstract diagrams (Tables and Graphs) were used with Organize problems. Concrete diagrams and Graph were used with Visualize problems to support visual search. These results suggest the possibility of scaffolding in diagram use, 
first by using more concrete diagrams and then progressing onto more abstract diagrams like graphs when more complicated inferences need to be made.

Table 1. Corresponding frequencies between problem types and kinds of diagrams

\begin{tabular}{lcccccc|l}
\hline Diagram & \multicolumn{7}{c}{ Problem type } \\
\cline { 2 - 8 } \multicolumn{1}{c}{ kind } & Change & Combine & Compare & Vary & Organize & Visualize & Total \\
\hline Picture & 3 & $\mathbf{1 1}^{* *}$ & $0^{*}$ & 10 & 2 & $0^{*}$ & $26^{*}$ \\
Concrete & $\mathbf{2 5}^{* * *}$ & $\mathbf{1 3}^{* * *}$ & 3 & $20^{*}$ & $0^{* *}$ & $\mathbf{8}^{* *}$ & $\mathbf{6 9}^{* * *}$ \\
Schematic & 4 & 3 & $0^{*}$ & 8 & 5 & $0^{*}$ & $20^{* *}$ \\
Line & 3 & 9 & 2 & 9 & $0^{* *}$ & 1 & $24^{*}$ \\
Number line & $0^{* *}$ & $1^{* *}$ & $\mathbf{1 5}^{* * *}$ & $\mathbf{4 4}^{* * *}$ & $0^{* *}$ & 2 & $\mathbf{6 2} * * *$ \\
Table & $0^{* *}$ & $0^{* *}$ & 1 & $2^{* * *}$ & $\mathbf{1 4}^{* * *}$ & 4 & $21^{* *}$ \\
Graph & $0^{* *}$ & $0^{* *}$ & 2 & $0^{* * *}$ & $\mathbf{1 5}^{* * *}$ & $\mathbf{7}^{* *}$ & $24 *$ \\
\hline Total & 35 & 37 & 23 & 93 & 36 & 22 & 246 \\
Mean & 5.00 & 5.29 & 3.29 & 13.29 & 5.14 & 3.14 & 35.14 \\
SD & 8.98 & 5.56 & 5.28 & 15.00 & 6.64 & 3.29 & 20.93 \\
$\chi^{2}(6)$ & 96.80 & 35.08 & 50.96 & 101.57 & 51.50 & 20.64 & 74.81 \\
$\boldsymbol{p}$ (adjusted) & 0.00 & 0.00 & 0.00 & 0.00 & 0.00 & 0.00 & 0.00 \\
\hline
\end{tabular}

$\boldsymbol{p}$ (adjusted): $\mathrm{p}$-value adjusted by Bonferroni method. ${ }^{*} \boldsymbol{p}<.05,{ }^{*} \boldsymbol{p}<.01,{ }^{* * *} \boldsymbol{p}<.001$. The asterisks indicate significantly higher (with bolded values) or lower proportions.

These results clarify the correspondence between problem types and kinds of diagrams as portrayed in Japanese textbooks. As this examination was conducted using only one of the book series for elementary schools, it needs to be conducted also for the other series/levels of education, and for textbooks in other countries to promote a better understanding of how textbooks contribute to the cultivation of competencies in diagram use. Future research will also need to scrutinize the efficacy of the kinds of diagrams indicated in textbooks for solving different types of problems.

Acknowledgment. This research was supported by a grant-in-aid (20K20516) received from the Japan Society for the Promotion of Science.

\section{References}

1. Daroczy, G., Wolska, M., Meurers, W.D., Nuerk, H.-C.: Word problems: a review of linguistic and numerical factors contributing to their difficulty. Front. Psychol. 06, 1-13 (2015)

2. Uesaka, Y., Manalo, E., Ichikawa, S.: What kinds of perceptions and daily learning behaviors promote students' use of diagrams in mathematics problem solving? Learn. Instr. 17, 322-335 (2007)

3. Ayabe, H., Manalo, E., Hanaki, N.: Teaching diagram knowledge that is useful for math word problem solving. In: EAPRIL 2019 Conference Proceedings, pp. 388-399 (2020) 
4. Souma,K., et al.: Tanoshii Sansuu 6 Nen (Fun Math Grade 6), 1st edn. Dainippon Tosho Publishing, Tokyo (2020)

5. Kintsch, W., Greeno, J.G.: Understanding and solving word arithmetic problems. Psychol. Rev. 92, 109-129 (1985)

Open Access This chapter is licensed under the terms of the Creative Commons Attribution 4.0 International License (http://creativecommons.org/licenses/by/4.0/), which permits use, sharing, adaptation, distribution and reproduction in any medium or format, as long as you give appropriate credit to the original author(s) and the source, provide a link to the Creative Commons license and indicate if changes were made.

The images or other third party material in this chapter are included in the chapter's Creative Commons license, unless indicated otherwise in a credit line to the material. If material is not included in the chapter's Creative Commons license and your intended use is not permitted by statutory regulation or exceeds the permitted use, you will need to obtain permission directly from the copyright holder.

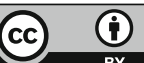

